\title{
Glycaemic response to barley porridge varying in dietary fibre content
}

\author{
Pariyarath S. Thondre*, Ke Wangt, Andrew J. Rosenthal and Christiani J. K. Henry \\ School of Life Sciences, Functional Food Centre, Oxford Brookes University, Oxford OX3 OBP, UK
}

(Received 17 January 2011 - Revised 24 March 2011 - Accepted 26 May 2011 - First published online 26 July 2011)

\begin{abstract}
The interest in barley as a food is increasing worldwide because of its high dietary fibre (DF) content and low glycaemic index (GI). DF in cereals may prove beneficial in improving blood glucose response in the long term. However, a dose-dependent effect of insoluble fibre on reducing postprandial blood glucose levels is yet to be proven. The objective of the present study was to determine the glycaemic response to two barley porridges prepared from whole barley grains varying in fibre content. In two separate non-blind randomised crossover trials, ten human subjects consumed barley porridge with $16 \mathrm{~g} / 100 \mathrm{~g}$ and $10 \mathrm{~g} / 100 \mathrm{~g}$ fibre content provided in different serving sizes (equivalent to 25 and $50 \mathrm{~g}$ available carbohydrate). The glycaemic response to both barley porridges was significantly lower than the reference glucose $(P<0 \cdot 05)$. There was no significant difference between the glucose areas under the curve or GI for the two barley porridges. We concluded that irrespective of the difference in total fibre content or serving size of barley porridges, their GI values did not differ significantly.
\end{abstract}

Key words: Barley: Dietary fibre: Glycaemic response

Barley is one of the oldest cultivated cereal grains in the world $^{(1)}$. In Western countries, barley is commonly used for feed, malt and alcohol production. Barley malt is used to enhance flavour in food products such as breakfast cereals and breads. In some regions, such as Tibet and Morocco, barley is consumed in large amounts. Barley is also used to make porridge, soup, snacks and beverages. The interest in barley as a food is increasing worldwide because of its high dietary fibre (DF) content and the recent approval of $\beta$-glucan health claims for cholesterol lowering by the United States Food and Drug Administration. It is now widely accepted that the $\beta$-glucans in barley foods are effective in lowering blood cholesterol ${ }^{(2)}$ and glycaemic response $(\mathrm{GR})^{(3,4)}$.

Whole barley grain consists of about $65-68 \%$ starch, $10-17 \%$ protein, $4-9 \% \beta$-glucan, $2-3 \%$ free lipids and $1.5-2.5 \%$ minerals $^{(5,6)}$. In addition, the total DF content ranges from 11 to $34 \%$ of which the soluble fibre content is between 3 and $20 \%{ }^{(7)}$. In addition to the high-fibre content, barley is also rich in phenolic compounds such as polyphenols, phenolic acids, proanthocyanidins and catechins. The international table for glycaemic index (GI) and glycaemic load values, 2008 has classified barley as the food grain with the lowest $\mathrm{GI}^{(8)}$.

The concept of GI was first introduced in 1981 as a means for identifying and classifying carbohydrate-rich foods based on their ability to raise postprandial blood glucose levels ${ }^{(9)}$. A low or attenuated GR is beneficial in both healthy and diabetic people. Low-GI foods have proven beneficial in the management of diabetes, obesity and $\mathrm{CVD}^{(10-12)}$. In GI testing, the test meal consumed is often equivalent to $50 \mathrm{~g}$ available carbohydrate and is compared with $50 \mathrm{~g}$ glucose as the reference food. However, in the case of foods having a low-to-moderate carbohydrate density, the quantity of available carbohydrate in the test portion can be reduced to avoid a large volume. It is recommended that the amount of available carbohydrate can range from 25 to $50 \mathrm{~g}^{(13)}$.

Epidemiological studies have suggested that consumption of fibre-rich foods can enhance satiety, improve blood glucose and cholesterol levels, and maintain healthy $\mathrm{BMI}^{(14,15)}$. The average recommended daily intake of DF is $25 \mathrm{~g} / \mathrm{d}$. Feeding studies using whole grain barley have been reported to enhance satiety ${ }^{(16)}$, reduce blood pressure ${ }^{(17)}$ and reduce serum cholesterol ${ }^{(18)}$, but there have not been many studies on GR to whole grain barley. The fibre content of barley can vary widely depending on the source and methods of milling. We hypothesised that a barley variety with a higher fibre content will have a lower GR. The objective of the present study was to determine the GR to two barley porridges prepared from whole barley grains with different fibre contents (barley 1 and barley 2) and provided in different serving sizes.

Abbreviations: DF, dietary fibre; GI, glycaemic index; GR, glycaemic response; iAUC, incremental area under the curve.

*Corresponding author: P. S. Thondre, fax +44 1865 483618, email pthondre@brookes.ac.uk

† Present address: Postgraduate Medical School, University of Surrey, Daphne Jackson Road, Manor Park, Guildford, UK. 


\section{Experimental methods}

\section{Materials}

The reference food glucose (dextrose monohydrate) was from Lloyds Pharmacy Limited (Coventry, UK). Pearl barley (barley 1) was from a local supermarket (Waitrose Limited, Bracknell, UK) and Suma Organic barley grain (barley 2) was from Suma Whole Foods (Elland, UK). The composition of these two types of barley is given in Table 1 .

\section{Determination of $\beta$-glucan content in barley grains}

The barley grains were milled using a Cyclotec ${ }^{\mathrm{TM}} 1093$ Sample Mill (FOSS in Britain and Ireland, Warrington, UK), with a $0.5 \mathrm{~mm}$ mesh screen. The $\beta$-glucan content in the milled barley flour was assayed using a Megazyme mixed-linkage $\beta$-glucan kit by hydrolysing with lichenase $(1000 \mathrm{U} / \mathrm{ml})$ and $\beta$-glucosidase $(40 \mathrm{U} / \mathrm{ml})$. The glucose produced was assayed against standard glucose using a glucose oxidase $(>12000 \mathrm{U}) /$ peroxidase $(>650 \mathrm{U}$ ) reagent by measuring the absorbance at $510 \mathrm{~nm}$ using a Shimadzu UV-1201 spectrophotometer (Shimadzu Scientific Instruments, Rydalmere, New South Wales, Australia).

\section{Subjects}

A total of twenty subjects (three men and seventeen women) from among the staff and students of Oxford Brookes University were recruited to participate in the study. The selection criteria were as follows: age 20-30 years, BMI between 18.5 and $25.0 \mathrm{~kg} / \mathrm{m}^{2}$, and fasting blood glucose value $<6.1 \mathrm{mmol} / \mathrm{l}$. All subjects were asked to fill a health questionnaire prior to the first test. Participants were given complete details of the study protocol and were given the opportunity to ask questions or withdraw from the study at any time. This study was conducted according to the guidelines laid down in the Declaration of Helsinki and all procedures involving human subjects were approved by the University Research and Ethics Committee at Oxford Brookes University. Written informed consent was obtained from all the subjects.

Anthropometric measurements were carried out for all the subjects using standardised methods, before the beginning of the study. Height was recorded to the nearest centimetre using a Stadiometer (Seca Limited, Birmingham, West Midlands, UK) with the subjects standing erect without shoes. Body weight was recorded using the Tanita BC-418 MA (Tanita UK

Table 1. Nutrition information of the barley grains used in the study*

\begin{tabular}{lrr}
\hline & Barley 1 & Barley 2 \\
\hline Energy $(\mathrm{kJ} / 100 \mathrm{~g})$ & 1533 & 1351 \\
Protein $(\mathrm{g} / 100 \mathrm{~g})$ & 9.9 & $8 \cdot 0$ \\
Carbohydrate $(\mathrm{g} / 100 \mathrm{~g})$ & $77 \cdot 7$ & 65.8 \\
Sugars $(\mathrm{g} / 100 \mathrm{~g})$ & $0 \cdot 8$ & $2 \cdot 1$ \\
Fat $(\mathrm{g} / 100 \mathrm{~g})$ & $1 \cdot 2$ & $2 \cdot 1$ \\
Saturates $(\mathrm{g} / 100 \mathrm{~g})$ & 0.2 & 0.3 \\
Fibre $(\mathrm{g} / 100 \mathrm{~g})$ & 15.6 & 9.6 \\
\hline * Information provided on the package.
\end{tabular}

* Information provided on the package.
Limited, Yiewsley, Middlesex, UK) with the subjects wearing light clothing and no shoes. BMI was calculated with the standard formula weight $(\mathrm{kg}) /$ height $\left(\mathrm{m}^{2}\right)$.

\section{Test meals}

The barley porridge was given in different serving sizes equivalent to either 25 or $50 \mathrm{~g}$ available carbohydrate. The energy and macronutrient composition of the test barley porridges are given in Table 2. Available carbohydrate was calculated for each test meal using the FAO/WHO procedure (total carbohydrate - DF) according to the nutrition information available from the manufacturers of barley grains. Those subjects ( $n$ 10) who consumed the $25 \mathrm{~g}$ serving size had $25 \mathrm{~g}$ glucose as the reference food and those subjects ( $n$ 10) who consumed the $50 \mathrm{~g}$ serving size had $50 \mathrm{~g}$ glucose as the reference food. Cooking time was according to the instructions on the packages, and the minimum advised cooking time was adjusted to both types of barley. Each sample was cooked separately in the morning when the subjects came for testing. Barley was weighed into a pan, washed thoroughly, brought to the boil with water and allowed to simmer until cooked. No salt or other ingredients were added into the barley porridge. The cooked porridge was served in a dish.

\section{Study design}

GR was measured and GI values of test meals were calculated using the FAO/WHO-recommended procedure ${ }^{(19)}$. All the foods were tested after a 12 -h overnight fast. As the subjects arrived in the morning, they were required to complete a small questionnaire regarding their last meal eaten, amount of alcohol and coffee consumed, exercise duration and level of stress. The reference food (glucose) and barley porridges were served with $200 \mathrm{ml}$ water and the subjects were required to eat at a comfortable pace within 10-12 min.

Two separate randomised, non-blind crossover trials were used to investigate the effects of two different serving sizes of barley 1 and barley 2 porridges on GR. The method of GI testing used in the study was adapted from the recommended GI methodology ${ }^{(13,20)}$. Five test sessions were involved in each study. Each subject had to test two test foods (barley 1 and barley 2 porridges) separately and the reference food

Table 2. Energy and macronutrient composition of barley 1 and barley 2 porridges in different serving sizes equivalent to 25 and $50 \mathrm{~g}$ available carbohydrate $(\mathrm{Av} \mathrm{CHO})$

\begin{tabular}{lccccc}
\hline & \multicolumn{3}{c}{ Barley type (Av CHO) } \\
\cline { 2 - 5 } & \multicolumn{2}{c}{ Barley 1} & & \multicolumn{2}{c}{ Barley 2 } \\
\cline { 2 - 3 } \cline { 5 - 6 } & $50 \mathrm{~g}$ & $25 \mathrm{~g}$ & & $50 \mathrm{~g}$ & $25 \mathrm{~g}$ \\
\hline Energy (kJ) & 1234 & 618 & & 1201 & 601 \\
Protein (g) & 8.0 & 4.0 & & 7.1 & 3.6 \\
Total CHO (g) & 62.5 & 31.3 & & 58.5 & 29.3 \\
Fat (g) & 1.0 & 0.5 & & 1.9 & 0.9 \\
Fibre (g) & 12.6 & 6.3 & & 8.5 & 4.3 \\
\hline CHO, carbohydrate. & & & &
\end{tabular}

$\mathrm{CHO}$, carbohydrate. 
(glucose) three times in a random order. The testing of each food was carried out on separate days and there was a gap of at least $1 \mathrm{~d}$ between each testing. According to the FAO/ WHO, six or more subjects are required in the repeated testing for determining the GI value of a food ${ }^{(19)}$. Therefore, ten subjects were recruited in each study, and all the tests were carried out in the morning, between 8.00 and 12.00 hours.

\section{Blood glucose measurements}

Subjects were encouraged to warm their hands to increase blood flow before taking the blood sample. Capillary blood glucose was analysed in finger-prick blood samples obtained using the Unistik 3 single-use lancing device (Owen Mumford, Woodstock, Oxford, UK). After discarding the initial two blood drops, the third drop was drawn into a HemoCue Glucose 201 microcuvette by capillary action. Glucose was measured by placing the microcuvettes in a HemoCue Glucose 201 + blood glucose analyser (HemoCue Limited, Dronfield, Derbyshire, UK). Fasting blood glucose measurement was carried out at 5 and 0 min before consumption of the test meal and the mean of both the values was used as the baseline blood glucose value. Further blood samples were taken after 15, 30, 45, 60, 90 and $120 \mathrm{~min}$ after the subjects started eating the test meal.

\section{Statistical analyses}

Statistical analysis was performed using Statistical Package for the Social Sciences (version 17.0; SPSS, Chicago, IL, USA). The intra-individual variations of the three reference glucose tests were assessed by determining the percentage of $\mathrm{CV}(\mathrm{CV} \%=100 \times \mathrm{sD} /$ mean $)$. The incremental areas under the curves (iAUC) were determined for blood glucose using the trapezoidal rule for values above the baseline and the paired $t$ test was used to assess the differences. All areas below the baseline were excluded from the calculations. GI was calculated from the iAUC with each subject being their own reference. Significant differences between the blood glucose values were evaluated by one-way ANOVA followed by Tukey's multiple comparisons test. Significance was set at $P<0.05$. Values are presented as means with their standard errors.

\section{Results}

All the twenty subjects completed the study. The baseline characteristics of the study population are given in Table 3 .
Data (mean and SD) for intra-individual variation in GR to the three reference tests were 19 (SD 13) \% CV for those subjects who consumed $25 \mathrm{~g}$ glucose and 25 (SD 10)\% CV for those subjects who consumed $50 \mathrm{~g}$ glucose. The two barley grains used to make the test porridges differed in their total fibre content but had very similar $\beta$-glucan content. Barley 1 had $16 \%$ fibre whereas barley 2 had $10 \%$ fibre. However, the $\beta$-glucan content was $3.5 \%$ in barley 1 and $3 \%$ in barley 2 .

Fig. 1 shows the GR to barley porridges containing $25 \mathrm{~g}$ available carbohydrates. There was a significant difference between the blood glucose values after the glucose reference and both barley 1 and barley 2 at 15, 30, 45, 60 and $120 \mathrm{~min}$ after consumption $(P<0 \cdot 05)$. Similarly, there was a significant difference in blood glucose after the consumption of the glucose reference and both barley 1 and barley 2 containing $50 \mathrm{~g}$ available carbohydrates $(P<0.05)$ at $15,30,45$ and 60 min (Fig. 2). A significant difference between only barley 2 and the glucose reference was observed at 90 min with the $50 \mathrm{~g}$ serving size $(P<0 \cdot 05)$, but there was no significant difference between the glucose reference and barley porridges at $120 \mathrm{~min}$ with the $50 \mathrm{~g}$ serving. There was no significant difference between the iAUC for the two barley porridges irrespective of the difference in serving sizes or the total fibre content (Fig. 3). The mean GI values of barley 1 were 44 (SEM 7) and 39 (SEM 10) with the 25 and $50 \mathrm{~g}$ available carbohydrate servings. This was not significantly different from the mean GI values of barley 2, which were 50 (SEM 5) and 43 (SEM 8) with the 25 and $50 \mathrm{~g}$ available carbohydrate servings.

The blood glucose values for barley 2 came down to the baseline quicker than barley 1 after the consumption of both serving sizes. The blood glucose values for barley 2 reached the baseline before $120 \mathrm{~min}$ in the case of the smaller serving size whereas for barley 1 , the blood glucose value continued above the baseline as is expected with higher fibre content. In the case of the larger serving size, the blood glucose levels did not reach the baseline in both barley 1 and barley 2 even after $120 \mathrm{~min}$. The blood glucose values for the reference food glucose reached almost near the baseline by 90 min after the $25 \mathrm{~g}$ serving size whereas the blood glucose values came near the baseline only by $120 \mathrm{~min}$ after the $50 \mathrm{~g}$ serving size.

\section{Discussion}

Pearl barley boiled for $60 \mathrm{~min}$ at a serving size containing $42 \mathrm{~g}$ available carbohydrate is reported to have a GI value of 35 $(\operatorname{sem} 4)^{(8)}$. The GI values obtained in the present study for barley 1 (38 (SEM 10)) and barley 2 (42 (SEM 7)) when served with $50 \mathrm{~g}$ available carbohydrate portions were not

Table 3. Baseline characteristics of the subjects who completed the study

(Mean values and standard deviations)

\begin{tabular}{|c|c|c|c|c|c|c|c|c|c|}
\hline \multirow[b]{2}{*}{ Subjects } & \multirow[b]{2}{*}{ Available $\mathrm{CHO}$ in the porridge $(\mathrm{g})$} & \multicolumn{2}{|c|}{ Age (years) } & \multicolumn{2}{|c|}{ Weight (kg) } & \multicolumn{2}{|c|}{ Height (m) } & \multicolumn{2}{|c|}{ BMI $\left(\mathrm{kg} / \mathrm{m}^{2}\right)$} \\
\hline & & Mean & SD & Mean & SD & Mean & SD & Mean & SD \\
\hline 10 (2 M; 8 F) & 25 & $23 \cdot 7$ & $1 \cdot 3$ & 54.4 & $6 \cdot 7$ & $1 \cdot 6$ & $0 \cdot 1$ & $20 \cdot 1$ & 1.6 \\
\hline $10(1 \mathrm{M} ; 9 \mathrm{~F})$ & 50 & $26 \cdot 3$ & $4 \cdot 5$ & $59 \cdot 9$ & $10 \cdot 4$ & $1 \cdot 7$ & $0 \cdot 1$ & $21 \cdot 2$ & $2 \cdot 3$ \\
\hline
\end{tabular}

$\mathrm{CHO}$, carbohydrate; $\mathrm{M}$, male; F, female. 


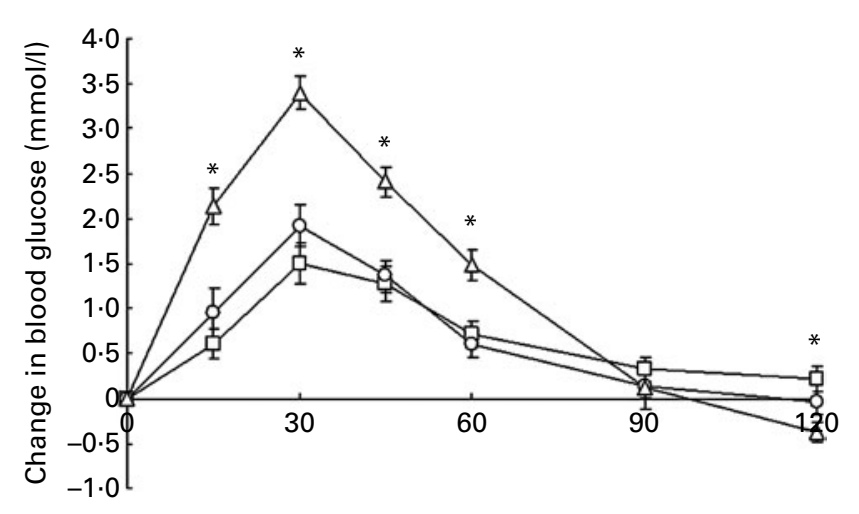

Time (min)

Fig. 1. Change in blood glucose after consumption of reference glucose $(\Delta)$, barley $1(\bigcirc)$ and barley $2(\square)$ porridges equivalent to $25 \mathrm{~g}$ available carbohydrate. Values are means, with their standard errors represented by vertical bars $(n 10)$. ${ }^{*}$ Mean values were significantly different $(P<0.05)$.

very different. There are only a few reports on GR studies using barley and most of them have used barley with other high- GI grains such as rice. The present study results were in agreement with a previous report, which found a significant reduction in plasma glucose levels when barley with $9 \cdot 2 \mathrm{~g}$ $\mathrm{DF} / 75 \mathrm{~g}$ available carbohydrate serving was compared with white rice and glucose $\mathrm{e}^{(21)}$.

The study ${ }^{(21)}$ rejected our hypothesis, as there was no significant difference between glycaemic responses to barley 1 and barley 2 porridges given in different serving sizes. This confirms that different serving sizes of test meals do not affect the overall iAUC or GI if compared with the same amount of reference glucose. However, it is interesting to note that the iAUC and GI for the larger serving size of both the barley porridges showed lower values although the differences were insignificant. This may be attributed to the higher fibre content in the larger serving size. The results of the present study contradict those presented in a previous study where a significant difference in glucose iAUC was noted after subjects consumed 25, 50 and $100 \mathrm{~g}$ carbohydrate

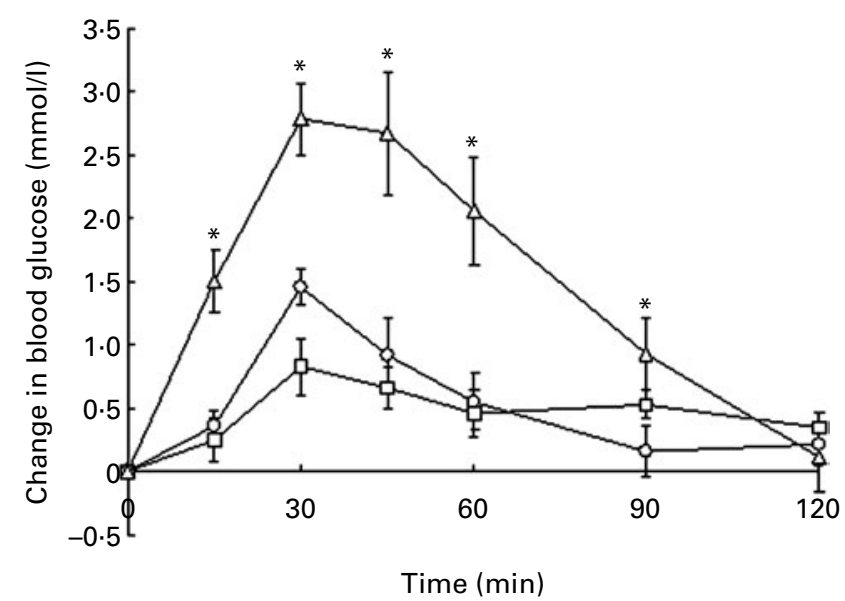

Fig. 2. Change in blood glucose after consumption of reference glucose $(\Delta)$, barley $1(\bigcirc)$ and barley $2(\square)$ porridges equivalent to $50 \mathrm{~g}$ available carbohydrate. Values are means, with their standard errors represented by vertical bars $(n 10) .{ }^{*}$ Mean values were significantly different $(P<0.05)$.

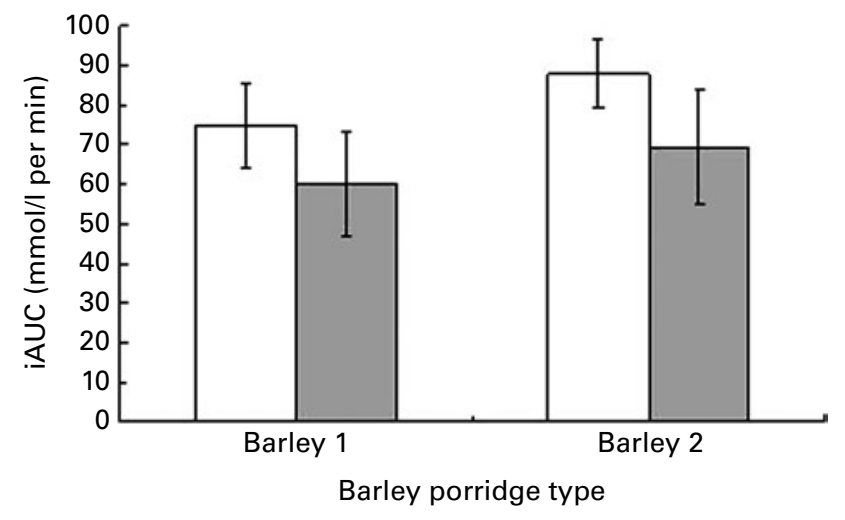

Fig. 3. Incremental area under the curve (iAUC) of barley 1 and barley 2 porridges tested as 25 and $50 \mathrm{~g}$ available carbohydrate serving sizes. ( $\square$ ), $25 \mathrm{~g}$ available carbohydrate serving; $(\square), 50 \mathrm{~g}$ available carbohydrate serving. Values are means, with their standard errors represented by vertical bars (n 10).

doses of pearl barley ${ }^{(22)}$. However, it may be noted that the relationship between the amount of carbohydrate and glucose responses was not linear. Furthermore, some subjects took more than $15 \mathrm{~min}$ to consume the 50 and $100 \mathrm{~g}$ barley meals, which might have resulted in increase in iAUC values.

Although the two barley grains used in this study were different in their total fibre content, their GI did not differ significantly. The reason for this could be the very similar $\beta$-glucan content in the two grains. Hence, it could be assumed that the soluble fibre content rather than total fibre content is more responsible for lowering GR in foods. The present study results were in agreement with a study that reported no difference in GR between a high-fibre cereal (33 g insoluble fibre) and a low-fibre cereal (1 g insoluble fibre ${ }^{(23)}$. However, the authors ${ }^{(23)}$ found lower blood glucose response following a second preset pizza meal after the highfibre treatment. This was attributed to the bacterial fermentation and production of SCFA. Hence, it may be assumed that in the present study as well that any effect of insoluble fibre would have become apparent only after a second meal.

The GR to both barleys were characterised by flattened peaks in comparison to the sharp rise in blood glucose values after consumption of the reference glucose drink. This is characteristic of low-GI foods. The glucose curves for barley 1 and barley 2 looked different in their shape with different serving sizes. However, the peak blood glucose values did not differ significantly. Recently, when GI values of foods were correlated to their postprandial GR, it was found that GI is highly correlated with the actual and incremental glucose concentrations at 60 and $90 \mathrm{~min}^{(24)}$. The present study results agree with this finding and show very similar actual glucose values for barley 1 at $60 \mathrm{~min}(5 \cdot 1$, $5.4 \mathrm{mmol} / \mathrm{l})$ and $90 \mathrm{~min}(5 \cdot 1,5 \cdot 0 \mathrm{mmol} / \mathrm{l})$ as well as for barley 2 at $60 \mathrm{~min}(5 \cdot 3,5 \cdot 1 \mathrm{mmol} / \mathrm{l})$ and $90 \mathrm{~min}(4.9,4.7 \mathrm{mmol} / \mathrm{l})$, thus resulting in no significant difference in GI.

Health benefits associated with whole-grain consumption have always been attributed to high levels of DF content in them. It is widely accepted that the viscous and gel-forming properties of soluble DF slow gastric emptying, nutrient 
absorption, and cholesterol and bile acid re-absorption in the gut to reduce postprandial glucose responses as well as total and LDL-cholesterol levels ${ }^{(25)}$. However, not all studies ${ }^{(26-31)}$ using isolated soluble DF have been able to prove this theory. At the same time, many studies ${ }^{(32-34)}$ have shown that the consumption of insoluble DF is associated with a reduced risk of type 2 diabetes. This could either relate to the SCFA production in the colon and subsequent effect on insulin sensitivity or to the presence of other beneficial compounds such as resistant starch and polyphenols associated with DF.

The present study was limited by the fact that barleys with two different fibre contents were only used without a dose-response study on total fibre content. Similar levels of $\beta$-glucan in the two barley grains also might have contributed to similar GR to barley 1 and barley 2 . It may be assumed that the combined effect of soluble and insoluble fibre is more important than just one present in high levels. A literature search reveals that there is very little difference between the GI values reported for whole-grain and refined-grain foods ${ }^{(8,35)}$. This confirms that the food matrix and physical structure of starchy foods are more important than just the fibre content in indicating the GI of foods. Although epidemiological studies have confirmed that increasing fibre intake can reduce the risk of diabetes, randomised controlled trials have not yet been successful in demonstrating the dose-dependent reduction of postprandial blood glucose levels by DF consumption.

To summarise, the present study compared the GR to two barley grains with different fibre content in different serving sizes. Although the GR to both barley grains were low, there was no difference in the GR to both barley grains irrespective of the difference in total fibre content or serving size. It may be concluded that a $6 \%$ difference in total fibre content does not have an influence on postprandial blood glucose and the effect may be more pronounced with differences in soluble fibre. The results presented reiterate the use of barley for the maintenance of healthy blood glucose levels. Further investigation is warranted to identify whether higher fibre content $(>16 \mathrm{~g} / 100 \mathrm{~g})$ will have an effect on postprandial blood glucose levels.

\section{Acknowledgements}

P. S. T. acknowledges financial support from the Higher Education Innovation Fund (HEIF) and K. W. acknowledges Oxford Brookes University Funding. The authors thank Megan Pratt for laboratory assistance and all the participants who took part in the study. P. S. T. designed and supervised the study and prepared the manuscript, K. W. carried out the study, A. J. R. helped K. W. in conducting the study and C. J. K. H. helped in the design, execution of the study and manuscript preparation. The authors have no conflict of interest.

\section{References}

1. Baik BK \& Ullrich SE (2008) Barley for food: characteristics, improvement and renewed interest. J Cereal Sci $\mathbf{4 8}$, $233-242$.
2. Behall KM, Scholfield DJ \& Hallfrisch J (2004) Diets containing barley significantly reduce lipids in mildly hypercholesterolemic men and women. Am J Clin Nutr 80, 1185-1193.

3. Cavallero A, Empilli S, Brighenti F, et al. (2002) High $(1 \rightarrow 3$, $1 \rightarrow 4) \beta$-glucan barley fractions in bread making and their effects on human glycemic response. J Cereal Sci 36, 59-66.

4. Thondre PS \& Henry CJK (2009) High-molecular-weight barley $\beta$-glucan in chapatis (unleavened Indian flatbread) lowers glycemic index. Nutr Res 29, 480-486.

5. Izydorczyk MS, Storsley J, Labossiere D, et al. (2000) Variation in total and soluble $\beta$-glucan content in hulless barley: effects of thermal, physical, and enzymic treatments. J Agric Food Chem 48, 982-989.

6. Quinde Z, Ullrich SE \& Baik BK (2004) Genotypic variation in colour and discolouration potential of barley-based food products. Cereal Chem 81, 752-758.

7. Fastnaught CE (2001) Barley fibre. In Handbook of Dietary Fibre, pp. 519-542 [S Cho and M Dreher, editors] New York: Marcel Dekker.

8. Atkinson FS, Foster-Powell K \& Brand-Miller JC (2008) International tables of glycemic index and glycemic load values: 2008. Diabetes Care 31, 2281-2283.

9. Jenkins DJA, Wolever TMS, Taylor RH, et al. (1981) Glycemic index of foods: a physiological basis for carbohydrate exchange. Am J Clin Nutr 34, 362-366.

10. Kalergis M, Grandpre ED \& Andersons C (2005) The role of the glycemic index in the prevention and management of diabetes: a review and discussion. Can J Diabetes 29, 27-38.

11. Jenkins DJA, Kendall CWC, Marchie A, et al. (2004) The glycaemic index: an overview of its possible role in the prevention and treatment of chronic disease. Int J Clin Pract 58, Suppl. 142, 3-7.

12. Marsh K \& Brand-Miller J (2008) State of the art reviews: glycemic index, obesity and chronic disease. Am J Lifestyle Med 2, 142-150.

13. Brouns F, Bjorck I, Frayn KN, et al. (2005) Glycaemic index methodology. Nutr Res Rev 18, 145-171.

14. Liu S, Willett WC, Manson JE, et al. (2003) Relation between changes in intakes of dietary fibre and grain products and changes in weight and development of obesity among middle-aged women. Am J Clin Nutr 78, 920-927.

15. Schulz M, Nothlings U, Hoffmann K, et al. (2005) Identification of a food pattern characterized by high fiber and low fat food choices associated with low prospective weight change in the EPIC-potsdam cohort. J Nutr $\mathbf{1 3 5}$, 1183-1189.

16. Schroeder N, Gallaher DD, Arndt EA, et al. (2009) Influence of whole grain barley, whole grain wheat, and refined rice-based foods on short-term satiety and energy intake. Appetite 53, 363-369.

17. Hallfrisch J, Scholfield DJ \& Behall KM (2003) Blood pressure reduced by whole grain diet containing barley or whole wheat and brown rice in moderately hypercholesterolemic men. Nutr Res 23, 1631-1642.

18. Ikegami S, Tomita M, Honda S, et al. (1996) Effect of boiled barley-rice feeding in hypercholesterolemic and normolipemic subjects. Plant Foods Hum Nutr 49, 317-328.

19. FAO/WHO (1998) Carbohydrates in Human Nutrition. Report of a Joint FAO/WHO Expert Consultation. Rome: WHO.

20. Wolever TMS, Jenkins DJA, Jenkins AL, et al. (1991) The glycaemic index: methodology and clinical implications. Am J Clin Nutr 54, 846-854.

21. Sakuma M, Yamanaka-Okumura H, Naniwa Y, et al. (2009) Dose-dependent effects of barley cooked with white rice 
on postprandial glucose and desacyl ghrelin levels. J Clin Biochem Nutr 44, 151-159.

22. Wolever TMS \& Bolognesi C (1996) Source and amount of carbohydrate affect postprandial glucose and insulin in normal subjects. J Nutr 126, 2798-2806.

23. Samra RA \& Anderson GH (2007) Insoluble cereal fibre reduces appetite and short-term food intake and glycemic response to food consumed 75 min later by healthy men. Am J Clin Nutr 86, 972-979.

24. Brand-Miller JC, Stockmann K, Atkinson F, et al. (2009) Glycemic index, postprandial glycemia, and the shape of the curve in healthy subjects: analysis of a database of more than 1000 foods. Am J Clin Nutr 89, 97-105.

25. Weickert MO \& Pfeiffer AFH (2008) Metabolic effects of dietary fibre consumption and prevention of diabetes. J Nutr 138, 439-442

26. Cugnet-Anceau C, Nazare JA, Biorklund M, et al. (2010) A controlled study of consumption of $\beta$-glucan enriched soups for 2 months by type 2 diabetic free-living subjects. Br J Nutr 103, 422-428.

27. Biorklund M, Holm J \& Onning G (2008) Serum lipids and postprandial glucose and insulin levels in hyperlipidemic subjects after consumption of an oat $\beta$-glucan-containing ready meal. Ann Nutr Metab 52, 83-90.

28. Chen J, He J, Wildman RP, et al. (2006) A randomized controlled trail of dietary fibre intake on serum lipids. Eur J Clin Nutr 60, 62-68.
29. Smith KN, Queenan KM, Thomas W, et al. (2008) Physiological effects of concentrated barley $\beta$-glucan in mildly hypercholesterolemic adults. J Am Coll Nutr 27, 434-440.

30. Li J, Kaneko T, Qin LQ, et al. (2003) Effects of barley intake on glucose tolerance, lipid metabolism, and bowel function in women. Nutrition 19, 926-929.

31. Keogh GF, Cooper GJS, Mulvey TB, et al. (2003) Randomized controlled crossover study of the effect of a highly $\beta$-glucanenriched barley on cardiovascular disease risk factors in mildly hypercholesterolemic men. Am J Clin Nutr $\mathbf{7 8}$, $711-718$.

32. Schulze MB, Schulz M, Heidmann C, et al. (2007) Fibre and magnesium intake and incidence of type 2 diabetes: a prospective study and meta-analysis. Arch Intern Med 167, 956-965.

33. Brownlee IA (2009) The physiological roles of dietary fibre. Food Hydrocoll 25, 238-250.

34. De Munter JS, Hu FB, Spiegelman D, et al. (2007) Whole grain, bran, and germ intake and risk of type 2 diabetes: a prospective cohort study and systematic review. PLoS Med 4, e261.

35. Foster-Powell K, Holt SHA \& Brand-Miller JC (2002) International table of glycemic index and glycemic load values: 2002. Am J Clin Nutr 76, 5-56. 